\title{
EL IMPACTO DE LOS MEDIOS DE TRANSPORTE EN LA MORFOLOGIA DE ANTOFAGASTA (CHILE) 1868-1892 ${ }^{1}$
}

\section{THE IMPACT OF THE MEANS OF TRANSPORTATION ON THE MORPHOLOGY OF ANTOFAGASTA (CHILE) 1868-1892}

\author{
A. Jostan Chaparro \& B. Handy Bravo \\ Universidad Católica del Norte, Chile. \\ Jostan.chaparro@ucn.cl \\ Handy.bravo@ucn.cl
}

\section{RESUMEN}

El desierto de Atacama, fue sin duda una zona de interés para inversores latinoamericanos y extranjeros que veían en sus minerales el potencial económico que solventaría la demanda internacional. Antofagasta fundada en 1868, fue un asentamiento que funcionó como nodo administrativo de las oficinas salitreras, y como puerta comercial del Océano pacífico con el desierto de Atacama. Lo que significó, ser una ciudad portuaria de primer orden en servicios y distribución comercial. Sin embargo, el encuentro de la actividad mercante, con la llegada del tren en 1873, repercutieron de manera abrupta y en un cambio sin precedentes en la relación puerto-ciudad. Por lo que se analizará mediante planos cartográficos situados entre 1866-1881, el fenómeno del impacto de los medios de transporte desde el modelo Anyport de Bird (1971) y reinterpretado por Rodrigue et al. (2006), todo a través del relato históricos descritos por Arce (1930).

Palabras clave: transformación urbana, Antofagasta-Chile, ferrocarril, auge salitrero, ciudad-puerto. Línea de investigación: Ciudad y Proyecto.

Tema: Morfología urbana.

\section{ABSTRACT}

The Atacama Desert was undoubtedly an area of interest for Latin American and foreign investors who saw in its minerals the economic potential that would meet international demand. Antofagasta founded in 1868, was a settlement that functioned as an administrative node for the nitrate offices, and as a commercial gateway from the Pacific Ocean to the Atacama desert. What it meant, being a port city of the first order in services and commercial distribution. However, the meeting of merchant activity, with the arrival of the train in 1873, had an abrupt impact and in an unprecedented change in the port-city relationship. Therefore, the phenomenon of the impact of means of transport will be analyzed using cartographic plans located between

\footnotetext{
${ }^{1}$ Este trabajo es parte del compendio de publicaciones de tesis: Relación puerto-ciudad en Antofagasta. Transformaciones morfológicas del borde histórico a partir del auge salitrero (S.XIX-XX), del Magister en Arquitectura en Zonas Áridas (MAZA) de la Universidad Católica del Norte, Antofagasta, Chile.
} 


\section{SÃOPAULO15 $\quad$ SA 17 LISBOA $25 \sim 26$ JUN 2020}

1866-1881, from the Anyport model by Bird (1971) and reinterpreted by Rodrigue et al. (2006), all through the historical account described by Arce (1930).

Keywords: urban transformation, Antofagasta-Chile, railroad, nitrate boom, city-port.

Research line: City and Project.

Topic: Urban morphology.

\section{La irrupción de los medios de transporte masivo en la Revolución industrial en el desierto de Atacama}

El impacto de la Revolución Industrial europea en la segunda mitad del siglo XIX llevó a América Latina a ser incorporada a los mercados mundiales, debido a la demanda de materia prima y alimentos, esto por el creciente aumento demográfico y expansión económica del Viejo Continente. Por lo que se requirió mejora en los medios de transporte y conexiones entre los centros de distribución y las zonas extractivas. En efecto, las ciudades tuvieron un acelerado proceso de urbanización, concentración demográfica y aumento económico sin precedentes, lo que impactó en la estructura y la forma urbana de las ciudades latinoamericanas. (Hardoy, 1974)

En este sentido, Luis Ortega ratifica que: "En el proceso de industrialización sudamericano, Chile se encontraba entonces en una situación particularmente favorable para participar de forma activa y derivar beneficios significativos de la coyuntura internacional expansiva". (1991, p.215)

Proceso tardío en Chile, impactó en tres áreas de cambio, el primero en 1840 con el arribo a Valparaíso de los vapores "Chile" y "Perú" de la Pacific Steam Navigation Company ${ }^{2}$, la segunda en el uso de la energía a vapor de propulsión interna utilizada en las maquinarias de la minería entre 1850 a 1880, por último, el uso temprano de esta energía en el uso del ferrocarril a partir de 1851. (Ortega, 1991)

En este sentido Ortega detalla:

"A partir de aquel año la construcción ferroviaria en el norte por parte del sector privado y en la región central por el Estado pasó a ser un importante elemento de modernización que junto con acortar distancias y reducir los costos de transporte, introdujo importantes elementos nuevos a la producción y los servicios: una nueva forma de organizar empresas, nuevo material de transporte y propulsión y un nuevo tipo de trabajador: conductores, ingenieros, maquinistas y mecánicos, junto con el personal administrativo, formaban un mundo nuevo estructurado en los terminales y a lo largo de la vía. Las líneas, locomotoras, coches de carga y pasajeros demandaron de instalaciones y personal para mano atención y reparación, más aún, cuando la expansión de la red fue relativamente rápida". (1991, p.214)

En definitiva, estos cambios y procesos no solo implantaron cambios en el accionar de las máquinas, sino que también trajeron nuevos requerimientos en el área laboral, demandando nuevos oficios y especialidades no existentes, que debían mantener el funcionamiento de la zona portuaria, las diversas maquinarias y las locomotoras, que durante el siglo XIX ya se entretejían en la actividad industrial en las ciudades.

${ }^{2}$ Empresa naviera que utiliza la navegación a vapor en el Pacífico. 


\section{SÃOPAULO15 17 LISBOA $25 \sim 26$ JUN 2020}

La zona del desierto de Atacama entre los paralelos $23^{\circ} \mathrm{S}$ al $25^{\circ} \mathrm{S}$ parte de Bolivia a inició del siglo XIX, y con posterioridad territorio del norte de Chile por la Guerra del Pacífico, fue una franja territorial desprovista de las condiciones mínimas para el habitar humano, limitado por la falta de agua potable y el abastecimientos de alimentos, pero que atrajo a inversores chilenos, bolivianos, latinoamericanos y europeos, que vieron en sus minerales el potencial económico que abastecería la demanda del comercio internacional. Este despliegue de transformaciones se vio reflejado en nuevas industrias y tecnologías, además de capital humano, que ayudó a planificar y organizar estas nuevas ciudades industriales, concebidas en el borde costero como puertos comerciales, y en el interior como las Company towns conocidas como oficinas salitreras, estableciendo un modelo económico urbano-territorial en base a la minería y sus servicios.

En relación a este fenómeno el Dr. Arquitecto Claudio Galeno expone:

"El desierto de Atacama fue incorporado a la globalización del mundo de siglo XIX gracias a los avances de la Segunda Revolución Industrial. Explorarlo significó levantar asentamientos en la adversidad de esos ambientes, requirió cartografiar el territorio y la aplicación de nuevas tecnologías como destiladoras solares, viaductos, hidroeléctricas y puertos, junto con el diseño de nuevas arquitecturas como hospitales y estaciones de ferrocarril." (2019, p.1)

En este sentido, cada avance tecnológico significó, inicialmente el poder habitar un desierto en condiciones mínimas, además de resolver y optimizar procesos requeridos por la industria extractiva. Sin embargo, no podemos dejar de enfatizar en uno de los hechos fundamentales para la aceleración de estos procesos, y es la aparición de la llegada y desarrollo de los medios de transporte a vapor, que marcaron un antes y después en la manera de concebir las ciudades durante el periodo de incorporación de las máquinas.

Y es en este cambio progresivo en la tecnificación de la ciudad, que desde el modelo Anyport planteado por James Bird en el crecimiento puerto-ciudad, que podemos contrastar los diversos cambios ocurridos previos, durante y posterior de la Revolución Industrial en las ciudades portuarias y de ello el desarrollo y la incorporación de las redes de transporte.

Inicialmente el modelo Anyport, cuenta con 6 etapas identificadas de desarrollo: I Puerto primitivo, II Extensión del muelle marginal (malecón), III Elaboración de muelles marginales (malecón), IV Época de elaboración del muelle, V Muelle lineal y VI Especialización de muelle, los que se desarrollan en 4 grandes etapas. Con posterioridad en 1971 el modelo Anyport, mantendría los cambios identificados del modelo anterior, sintetizando solo en 3 grandes estadios de desarrollo (ver fig.1) 


\section{SÃOPAULO15 17 LISBOA $25 \sim 26$ JUN 2020 \\ Seminário Internacional de Investigação em Urbanismo \\ Seminario Internacional de Investigación en Urbanismo}

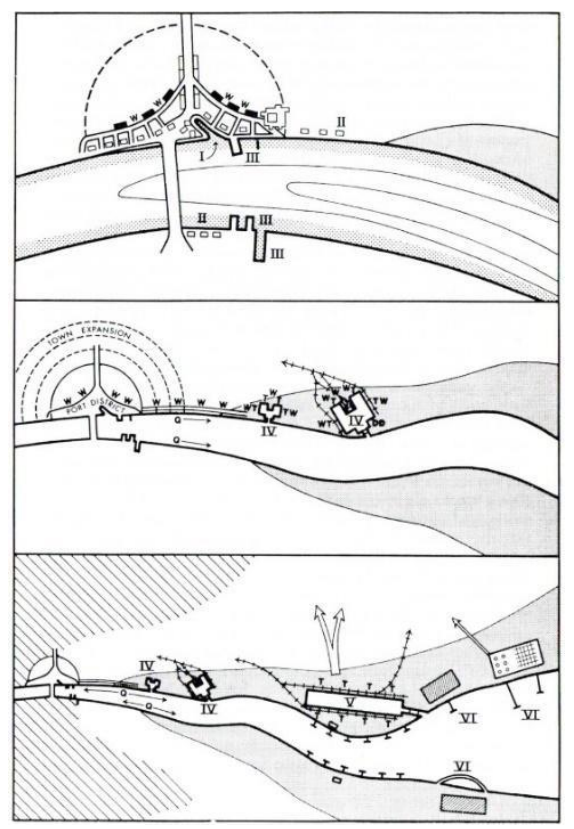

Fig. 1 Modelo Anyport. Fuente: (Bird, J. 1971).

Y es a partir de este principio que los doctores Jean-Paul Rodrigue, Claude Comtois y Brian Slack (2006) hacen una reinterpretación del modelo de Bird (1971) renombrando a estas 3 grandes etapas en establecimiento, expansión y especialización. (ver Fig. 2)
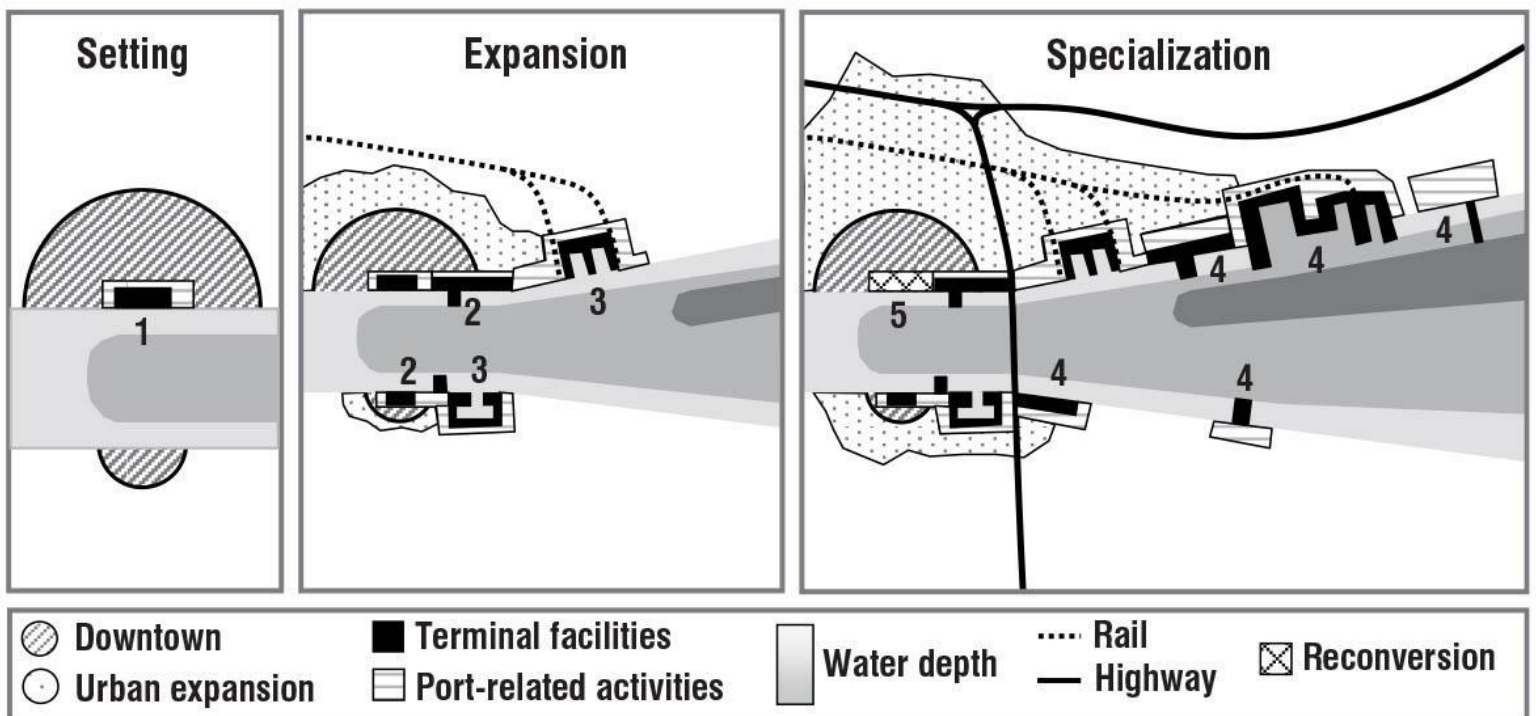

Fig. 2 modelo Anyport de Bird (1971) reinterpretado por Jean-Paul Rodrigue, Claude Comtois and Brian Slack. (2006) Fuente: The Geography of Transport. 


\section{SÃOPAULO15 17 LISBOA $25 \sim 26$ JUN 2020}

En la primera interfaz evolutiva llamada "establecimiento": el puerto se sitúa frente al núcleo urbano central, donde se encuentran las autoridades portuarias, las casas de comerciantes y los equipamientos en la línea costera. Este proceso ocurre anterior a la Revolución Industrial, periodo donde los puertos eran rudimentarios y se basaban en las actividades principalmente en la pesca, comercio y la construcción naval, que incluye la construcción de varios muelles.

En una segunda fase, denominada "expansión": comenzó con la llegada de la Revolución Industrial, cuando se desencadenaron varios procesos que afectaron a las actividades portuarias. Los muelles se extendieron debido a la creciente cantidad de carga y el tamaño de los barcos (2), necesitando la construcción de más muelles para sobrellevar la creciente demanda (3), el ferrocarril fue incorporado al terminal portuario dando más accesibilidad e influencia a mayores zonas.

Por último, en la etapa de mayor desarrollo conocida como "especialización": considerado un periodo postindustrial, se destaca por iniciar con la construcción de muelles especializados en contenedores, minerales, petróleo y carbón (4) la llegada de barcos más grandes requirió de dragados o construcciones fuera del núcleo central. Además, los puertos originales en esta etapa quedaron obsoletos y abandonados. Abriendo la posibilidad de reconversión de usos públicos (5).

En referencia a los descrito, el modelo Anyport, no es un prototipo único restrictivo para una ciudad puerto, sino que proporciona una base comparativa referencial de lo que podría evolucionar un puerto a través del progreso tecnológico, temporal y espacial, por medio de un modelo abstracto. Del que nos centraremos en las dos primeras etapas, establecimiento y expansión, debido a los parámetros del estudio y el análisis de cambio.

\section{Establecimiento: el surgimiento de la ciudad industrial puerto de Antofagasta.}

El puerto de la Chimba $^{3}$ ciudad portuaria del norte de Chile, se sitúo entre el desierto de Atacama y el Océano Pacífico, naciendo como un artificio que buscaba habitar lo inhóspito del desierto y sus carencias para la vida. Este lugar no presentaba antecedentes coloniales, ni un pasado que le presidiera, solo algunos vestigios de nómades que habitaron en base a la pesca, conocidos como los "changos" o "camanchacos", ubicados en distintos puntos de la costa, siendo un área relevante de restos arqueológicos, el sector de la Isla Guamán y sus alrededores, al norte de la ciudad. (Chervin, 1902)

La riqueza mineral hallada en el Salar del Carmen, descubierta por los ingenieros franceses, los hermanos Latrille ${ }^{5}$ asociados con M. Meunier en 1857, y explorada años más tarde por José Santo Ossa (Bermúdez, 1963, p.183), fue adjudicada en conjunto con Francisco Puelma ${ }^{6}$ en septiembre de 1866, cuya extensión abarcó $116 \mathrm{~km}^{7}$ cuadrados continuos, para la explotación del salitre y bórax en los terrenos descubiertos. En

\footnotetext{
${ }^{3}$ Nombre con el cual coexistió Antofagasta en sus primeros años.

4 Los Changos fueron pescadores, descendientes de una larga tradición de culturas dedicadas a la pesca y explotación de los recursos marinos, que habitaron la costa del norte de Chile

5 Domingo Latrille Loustauneau, ingeniero francés, fundador de la ciudad de Tocopilla, y hermano de Máximo Latrille Loustauneau, fueron los primeros exploradores de salitre en la zona, siendo estos muy influyentes en los inicios del Boom salitrero.

6 Ingeniero chileno, socio de José Santo Ossa, quien en 1866 organizó la "Sociedad Exploradora del Desierto de Atacama"

7 cinco leguas cuadradas
} 


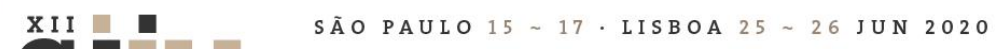

consecuencia, se requirió de la construcción de un puerto y un asentamiento minero frente al mar, fundándose el puerto de Antofagasta el 22 de octubre 1868.

En el plano para la futura población, diseñado por José Santo Prada en 1869 (Ver fig.03) se trazó un damero español, como la establecía las Leyes de Indias, curiosamente en un periodo tardío en plena segunda mitad del siglo XIX. Este fue orientado en $45^{\circ}$ respecto a los puntos cardinales, conformado principalmente por manzanas de $100 \times 100$ m, y manzanas irregulares dispuestas en el borde costero, ordenados en torno a una plaza central y un centro cívico. Presentándose 5 tipologías de subdivisión de manzanas: tipo A en 10 subdivisiones; tipo $B$ en 12 subdivisiones; tipo $C$ que correspondía a la mitad de la manzana subdivida en 5 áreas; tipo $D$ en dos subdivisiones seccionado por un pasaje, y finalmente el tipo $E$, que si bien no corresponde a una subdivisión es una manzana irregular adaptada al territorio del borde costero

Un elemento que se destaca en contraste a la proyección de las manzanas, es la superficie establecida para el terreno de la Compañía Melbourne Clark y Cía., que equivalía al 41\% (134.384 m2) en relación del total de sitios proyectados, siendo la superficie restante de $59 \%(189.845 \mathrm{~m} 2)$ dispuesta para viviendas, equipamientos, industrias y otros.

En este primer esbozo de esta nueva ciudad, se observa con claridad el valor de la industria frente a el área destinado a otros usos, donde el principal y hegemónico motor de desarrollo era potenciar la industria minera, siendo este el elemento principal de ordenamiento urbano que determinaría el devenir durante la época salitrera. En la zona del borde costero, se identifican tres muelles, los que corresponderían a proyecciones de la futura zona portuaria, de los que se contaba solo con la construcción de dos muelles, el primero llamado muelle de pasajeros o fiscal, recibido de manera oficial por el Perfecto de Cobija en 1868, construido por la Casa Melbourne Clark, en virtud a los compromisos pactados con el Gobierno boliviano. El segundo, de acuerdo con Contador (1982), fue construido en 1880, en remplazo al ya existente de 1869. A lo que le proseguirían diversos muelles hasta principio del siglo XX. 


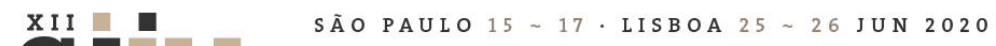

Seminário Internacional de Investigação em Urbanismo

Seminario Internacional de Investigación en Urbanismo

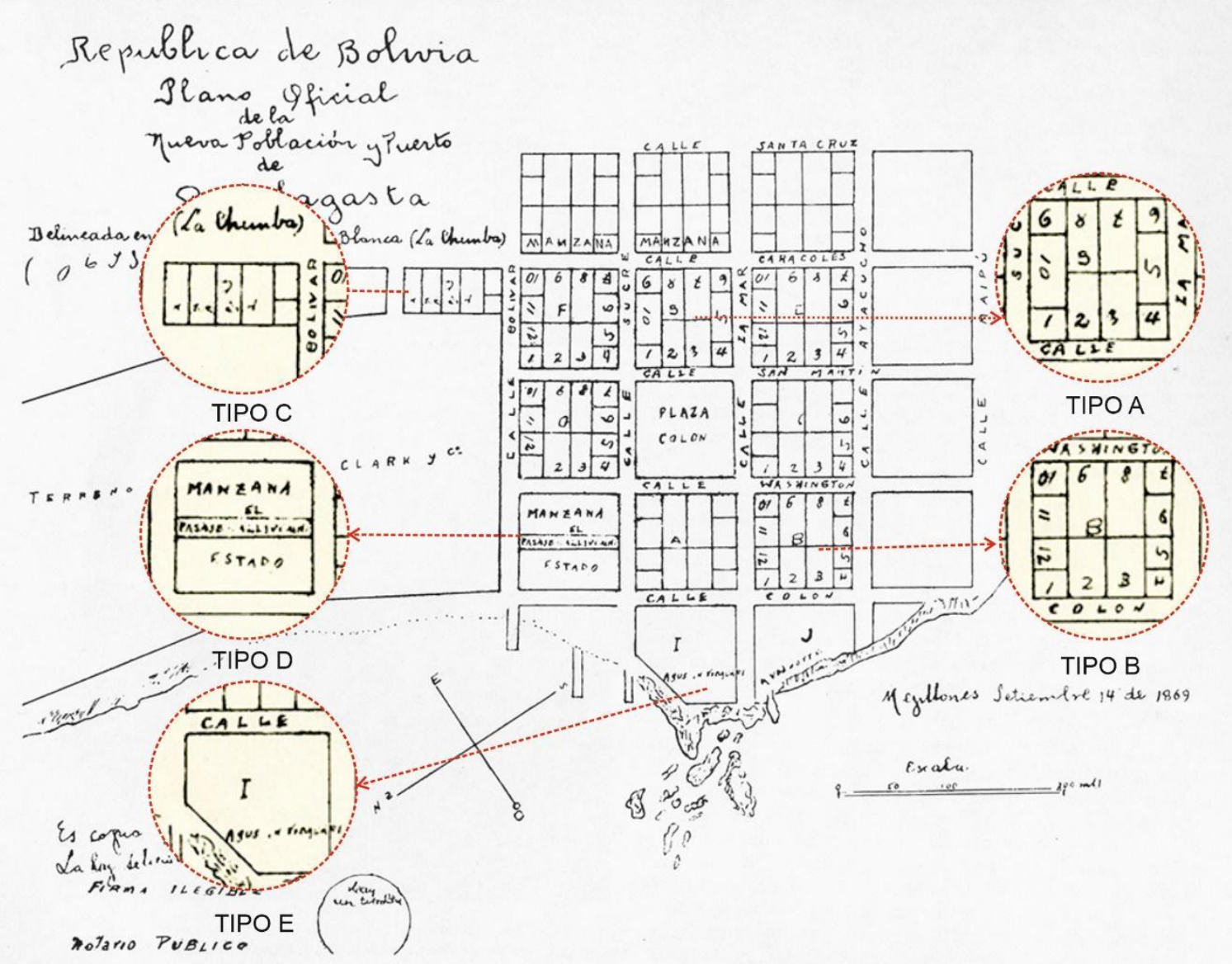

Fig. 3. Elaboración propia sobre el primer plano de Antofagasta elaborado por José Santos Prada y fechado en Mejillones en el año 1869. Fuente: Agullo, Antofagasta, una ciudad heroica, 1979.

Para 1870, gracias al descubrimiento de plata en la zona de Caracoles, el puerto de la Chimba ascendió a puerto menor en 1871 , hecho que significaría el abrir sus rutas comerciales a aguas internacionales, lo que se tradujo en mayores actividades comerciales en la costa, trayendo progreso y bonanza económica.

Un momento clave en la consolidación de los medios de transporte a nivel local y nacional fue 1872, por la conformación de la "Compañía de Salitres y Ferrocarril de Antofagasta", antes Compañía Melbourne Clark y Cía., quien gracias a los acuerdos económicos y permiso de explotación del salitre buscaron construir un tren para acelerar los procesos y reducir los costos de carga. Así también de manera paralela, surgió la "Compañía Sud Americana de Vapores (CSAV)" en Valparaíso, la cual se estableció en el puerto de esta ciudad, prestando servicios de cabotaje, conectando los diversos puertos nacionales y extranjeros, siendo Antofagasta muy favorecida de sus rutas marítimas. En este sentido Delgado afirma que: 


\section{SÃOPAULO15 $\quad$ SA 17 LISBOA $25 \sim 26$ JUN 2020}

"Las ciudades que más beneficio obtuvieron de la modernización de las infraestructuras de transporte fueron, sin lugar a dudas, las ciudades portuarias, que funcionaron como nodos intermodales en los que tenía lugar la ruptura de carga. No puede olvidarse, bajo este enfoque, que la mayor parte de las solicitudes y concesiones de construcción de caminos de hierro tuvieron el propósito de enlazar con los puertos para, desde ellos, exportar materias primas y productos elaborados (...)". (Delgado, 2010, p.1)

Y es en este rol de nodo entre el desierto Atacama y el Océano Pacífico, que la integración entre puerto y el ferrocarril fue fundamental para consolidar la industria minera. Teniendo como consecuencias en la ciudad, el surgimiento de límites, cambios morfológicos y grandes zonas industriales en el espacio urbano.

\section{Expansión: la integración del ferrocarril en la ciudad, el puerto y el territorio}

La zona portuaria de Antofagasta absorbió la actividad minera de dos Cantones ${ }^{8}$ salitreros, el primero de ellos fue el cantón central o boliviano, ubicado en el paralelo $23^{\circ}$, el que se ubicaba entre la zona de Baquedano hasta Sierra Gorda, conformado por 28 oficinas salitreras, siendo el puerto principal el de Antofagasta. El segundo llamado cantón de Aguas Blancas, se ubicó entre los paralelos $23^{\circ}$ y $24^{\circ}$, organizándose en 28 oficinas salitreras, que desembocaron en el puerto de Coloso, ubicado a $15 \mathrm{~km}$ al sur de Antofagasta. (Gonzales, 2008)

En esta etapa de desarrollo de la ciudad como puerto mercantil, irrumpió el ferrocarril, el cual inició el 13 de abril de 1872, gracias a la recién conformada empresa "Compañía de Salitres y Ferrocarril de Antofagasta", en solicitud para utilizar líneas férreas por algunas calles de la ciudad hasta su salida con la pampa, siendo terminadas el $1^{\circ}$ de diciembre de 1873. Durante la etapa de construcción y previo a la llegada de la locomotora, las vías fueron utilizada por carros tirados por dos yuntas de bueyes acarreando materiales, carbón, forraje, etc. Debido a la propicia pendiente y al magnífico cálculo de diseño del ingeniero Josiah Harding, estos carros cargados de salitre a granel se tiraban desde la cuesta solo con palanqueros encargados de controlar el descenso, cargamento que era vaciado frente a las costas del puerto. Harding había hallado una pendiente continua, sin desniveles, por la Cordillera de la Costa.

Sin embargo, esta condición sin el uso de una máquina a vapor no perduró, presentando de manera oficial el 20 de diciembre la primera locomotora en la ciudad, la que logró llegar hasta el establecimiento del Salar del Carmen.

En el Plano de Antofagasta de 1873 realizado por Adolfo Palacios, se logra apreciar el diseño de esta vía férrea, la que inicia en el terreno de la Compañía Melbourne Clark y Cía. en una curvatura simple, ingresando por el centro cívico y el área comercial de la ciudad, en un movimiento de zigzag, hasta atravesar hasta la plaza municipal donde se prolonga a través de ella hacia la pampa. (ver fig.04)

\footnotetext{
${ }^{8}$ División territorial que se hacía geográficamente a los terrenos salitrales, siempre en referencia a su medio de comunicación portuaria para los embarques. (Bahamondes, 1978, p.83)
} 


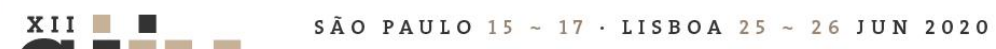

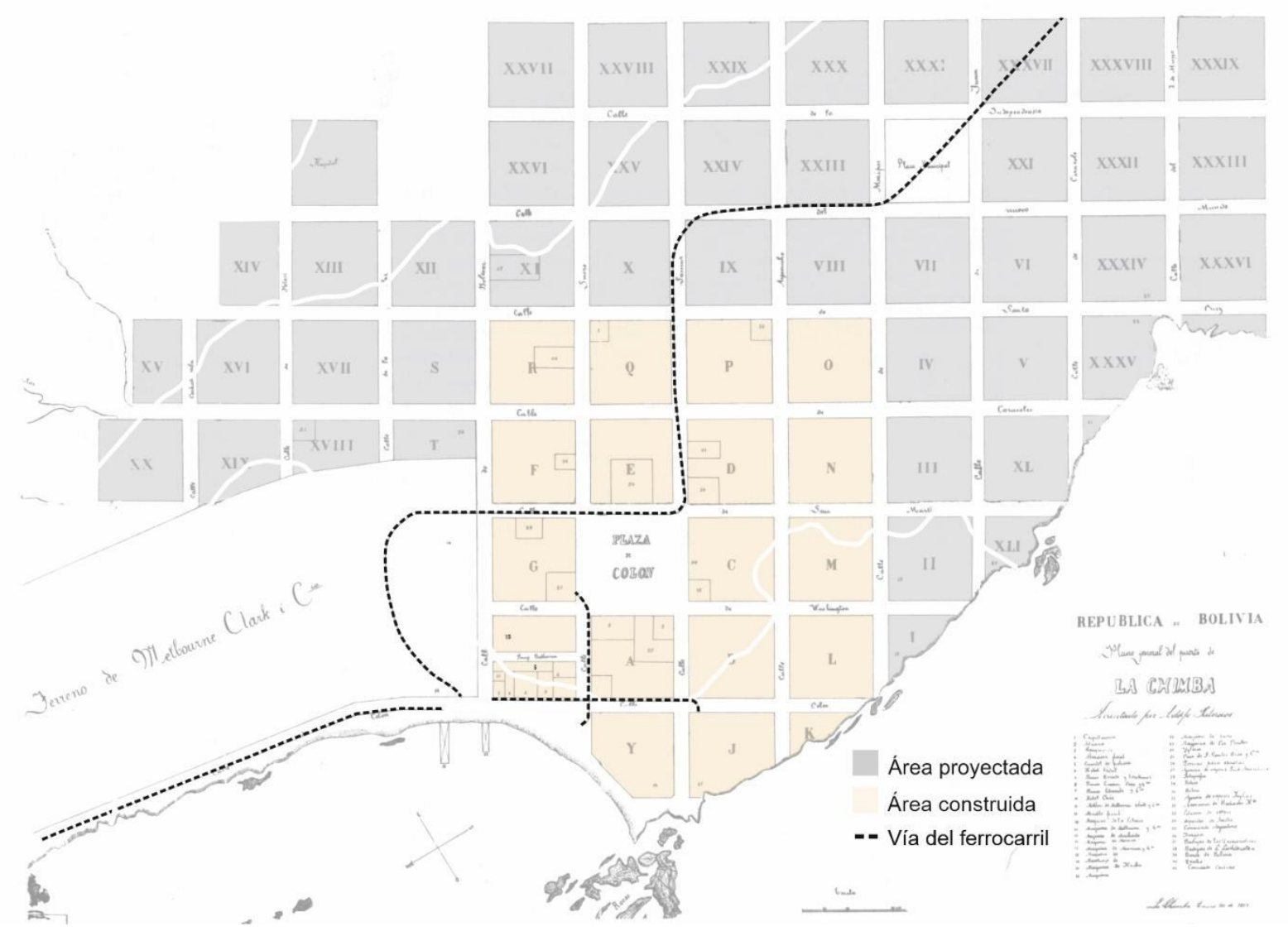

Fig. 4. Plano de Antofagasta 1873 realizado por Adolfo Palacios publicado por Agullo (1979). Fuente: Elaboración propia.

Arce señala, que el transitar del ferrocarril por el núcleo central de la ciudad, generó algunos incidentes ocasionados por las chispas que estas desprendían. A esto agrega el accidente de una locomotora en pleno centro de la ciudad, en la intersección entre la calle comercial (calle Prat) y calle Nuevo Mundo (actual calle Matta), donde murió el maquinista y el fogonero, debido la explosión de la caldera de la locomotora y en la que niños resultaron quemados.

Es así, que años más tarde y con un flujo constante de tráfico del ferrocarril por la ciudad, la empresa decidió trasladar esta vía inserta en el núcleo central, hacia la periferia (actualmente Avda. Argentina), para evitar accidentes. (Arce,1930)

En 1877, el uso del tren dejaría ser de uso exclusivo minero, iniciando el 18 de octubre el servicio público de pasajeros, aprobados por el gobierno boliviano frente a la necesidad de movilidad de los campamentos mineros. Este mismo año, el ingeniero Harding publicó en Londres un artículo en el Journal of the Royal Geographical Society, donde presentó un mapa del desierto de Atacama, destacándolos depósitos de nitrato encontrados hasta ese momento. En este mapa territorial, se logra observar a una escala mayor la ramificación de las vías del tren insertadas por el desierto, las que conectaba las distintas oficinas salitreras con las rutas hacia las ciudades portuarias, presentes en esta zona del norte de Chile. (ver fig.05) 


\section{XII $\square$ SÃO PAULO15 17 LISBOA $25 \sim 26$ JUN 2020}

Seminário Internacional de Investigação em Urbanismo

Seminario Internacional de Investigación en Urbanismo

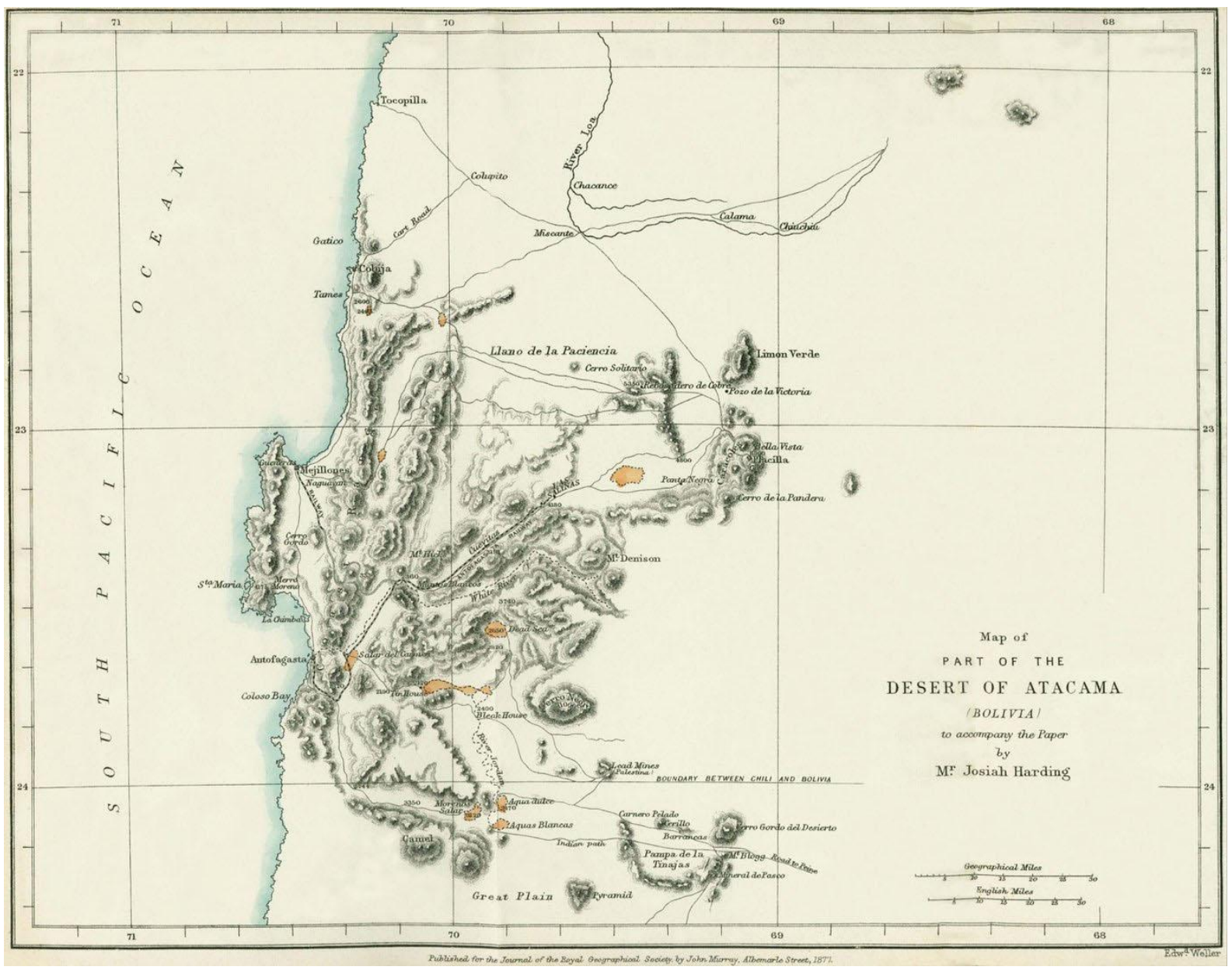

Fig. 5. Mapa de las riquezas del salitre del desierto de Atacama (Bolivia) publicado en 1877 por el ingeniero inglés Josiah Harding en la revista londinense Journal of the Royal Geographical Society.

Pese a desatarse en 1879 la Guerra del Pacífico, tensionado por el conflicto de impuestos y tratados no cumplidos, las nuevas rutas hacia el interior de la Pampa se mantuvieron.

El plano realizado por Vidal Gormaz en 1880 (Archivo BNC), hace evidente estos cambios urbanos morfológicos, evidenciado la tensión del circuito ferroviario enfrentado a la ortogonalidad diagonal de la trama urbana. Este proceso de superposición y direccionamiento segmentó la retícula urbana en cuatro áreas (Ver. Fig.6). La primera que definiremos como zona A, abarcaba de la plaza central hacia la costa y el lado sur de la ciudad, con manzanas planificadas (dibujadas con el centro vacío). En el costado oriente, una zona B, parte del actual centro histórico y las nuevas manzanas existentes contenidas por una nueva vía transversal que limita el crecimiento hacia la ladera del cerro, dejando afuera del tejido urbano al hospital y cementerio. En la zona C, se entrelazan las vías debido a que en esta área se posiciona la zona de acopio y centro de la Compañía Salitrera, siendo este el nodo intermodal de los trenes, donde se distribuía la carga hacia la zona portuaria y las fundiciones. En la parte oriente de esa área industrial se puede apreciar una planificada área de manzanas, (actual barrio Estación) contenida por la zona de la compañía salitrera y el camino de mulas 


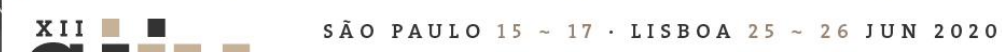

Seminário Internacional de Investigação em Urbanismo

Seminario Internacional de Investigación en Urbanismo

hacia el yacimiento de Caracoles. Al igual que en las zonas anteriores se muestra un Lazareto fuera del radio urbano y un fuerte en el extremo norte.

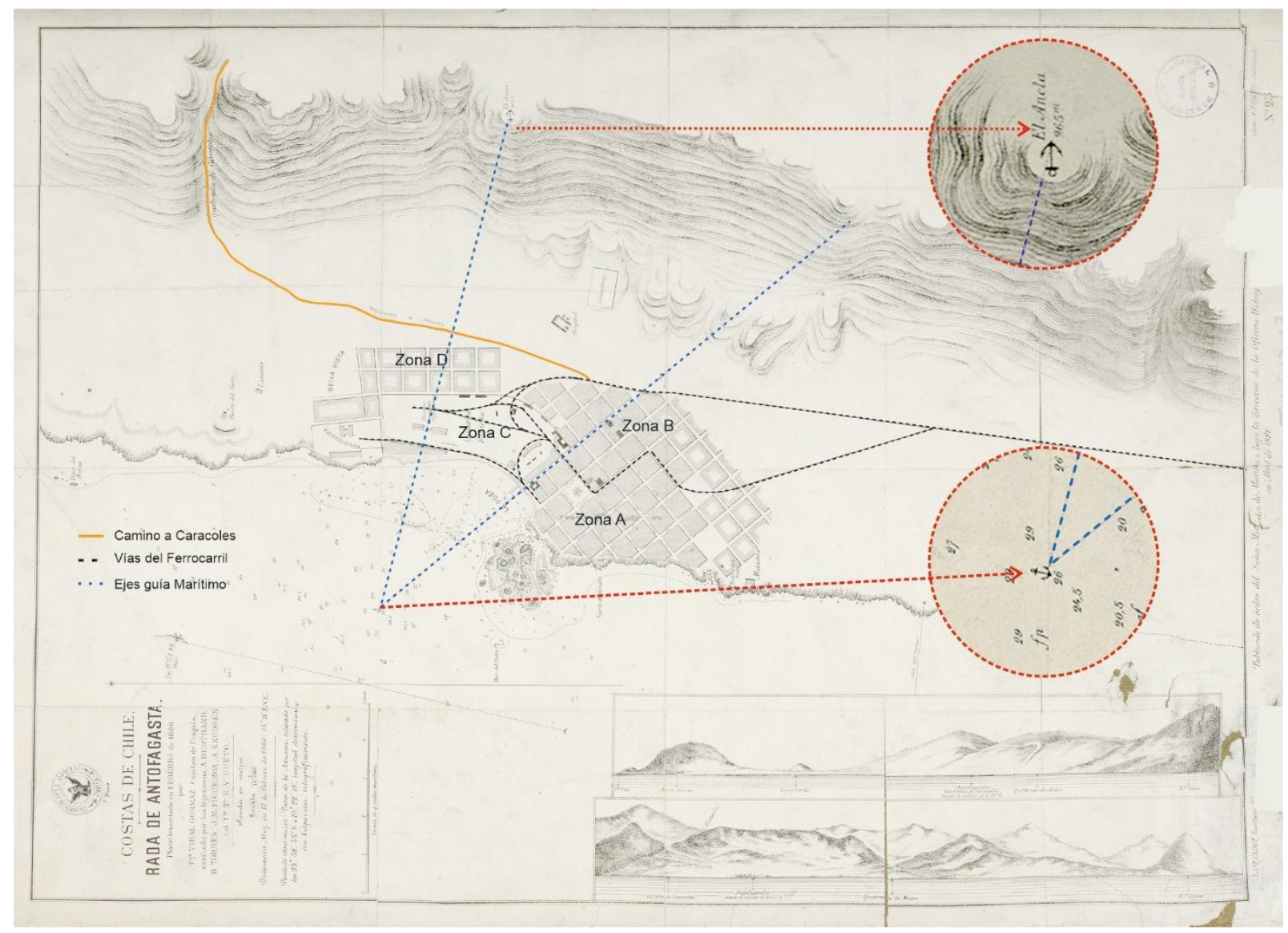

Fig. 06 La Poza con la batimetría, rocas y geometría del fondeadero en detalle del plano "Rada de Antofagasta" realizado en 1880 por Francisco Vidal Gormaz et al. Publicado en 1881. Archivo BNC. Fuente: Elaboración propia.

Así como las vías del tren son evidentes en el registro cartográfico, la rada del fondeadero de la Poza Histórica también era una guía para las embarcaciones y el desplazamiento de lanchones y botes, hacia las aguas de menor profundidad de la zona portuaria. Las que fijadas en el punto 26 , señalizada con un ancla, se proyectan en un ángulo de $56^{\circ}$ hacia dos ejes estratégicos de la ciudad, el primero hacia el cerro, sitio donde el ingeniero británico Jorge Hicks había dibujado un ancla en la cumbre de una montaña, y un segundo punto, en la proyección de la calle Bolívar, límite sur del patio industrial de la Compañía de Salitre, donde se ubicaba el muelle de pasajeros.

Mediante la demanda aumenta por la exportación de salitre, la construcción de muelles en la zona portuaria se masifico. En 1881 se llevó la incorporación de un nuevo muelle llamado Miraflores, su construcción fue en base a madera y fierro, teniendo en su diseño inicial una extensión de $100 \mathrm{~m}$. por $9.14 \mathrm{~m}$ de ancho ${ }^{9}$. Así

\footnotetext{
90 pies de ancho
} 


\section{SÃOPAULO15 17 LISBOA $25 \sim 26$ JUN 2020}

mismo un año después se inició la construcción del muelle Bellavista, el cual se aprobó el 27 de mayo de 1882, en respuesta a la solicitud enviada por la Casa de Jorge Brownell y Cía. Dueños de la fundación Bellavista. Teniendo como condicionante el estar habilitado para el embarque y desembarque por parte del Fisco. Además, ser el muelle auxiliar al muelle de pasajeros no pudiera cumplir el servicio por reparaciones. (Contador, 1982).

En su etapa de mayor crecimiento, el mar se densificó de extensiones de madera y acero, apropiándose del borde costero, constituyendo a mediados de los años 20' su mayor esplendor portuario, donde las actividades del puerto y la ciudad estaban unidas en torno al mar. (ver fig.07)

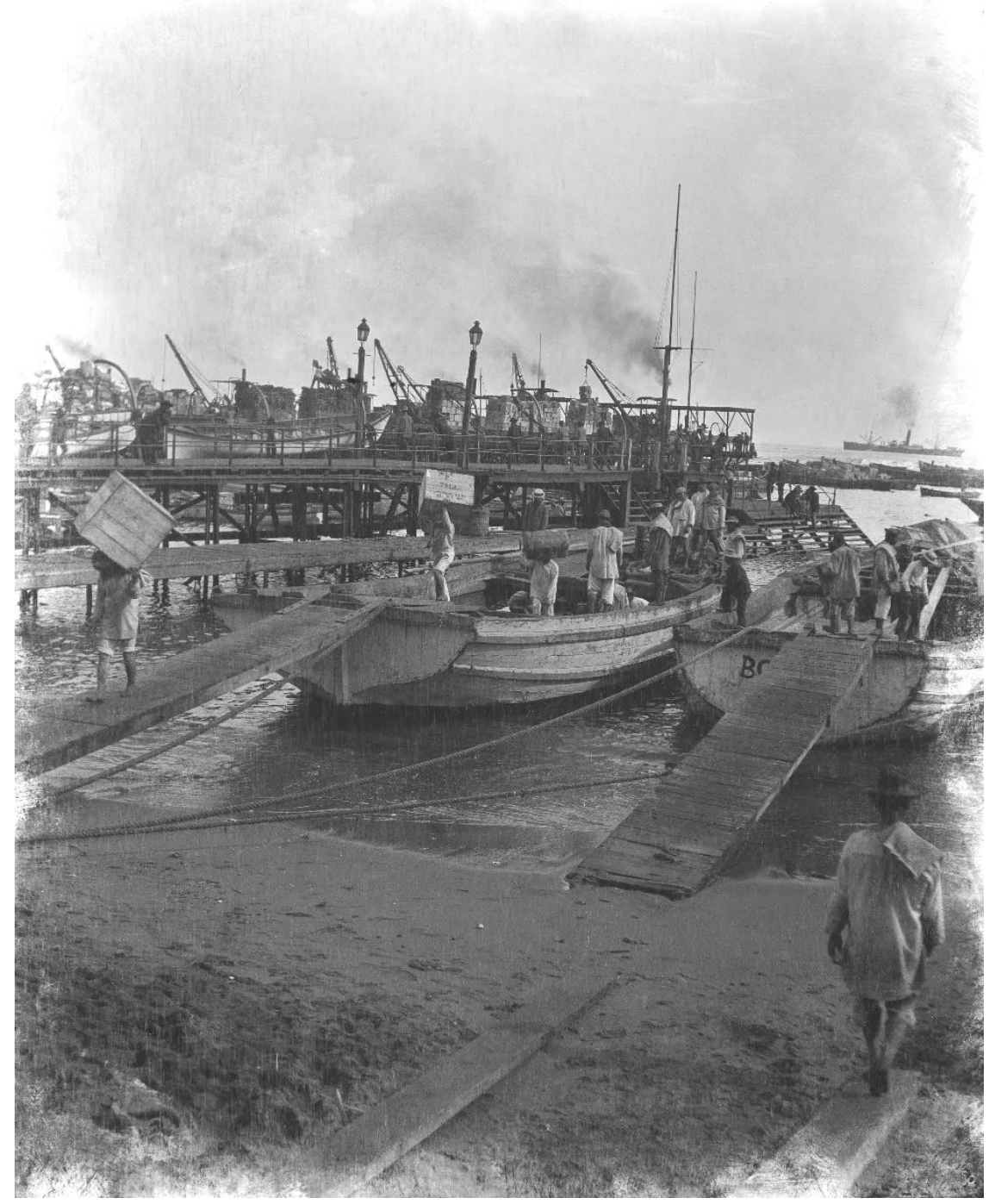

Fig. 07 Bowman, Isaiah, (C.1907-06) Antofagasta (Chile), men at harbor dock. Fuente: American Geographical Society Library, University of Wisconsin-Milwaukee Libraries. 


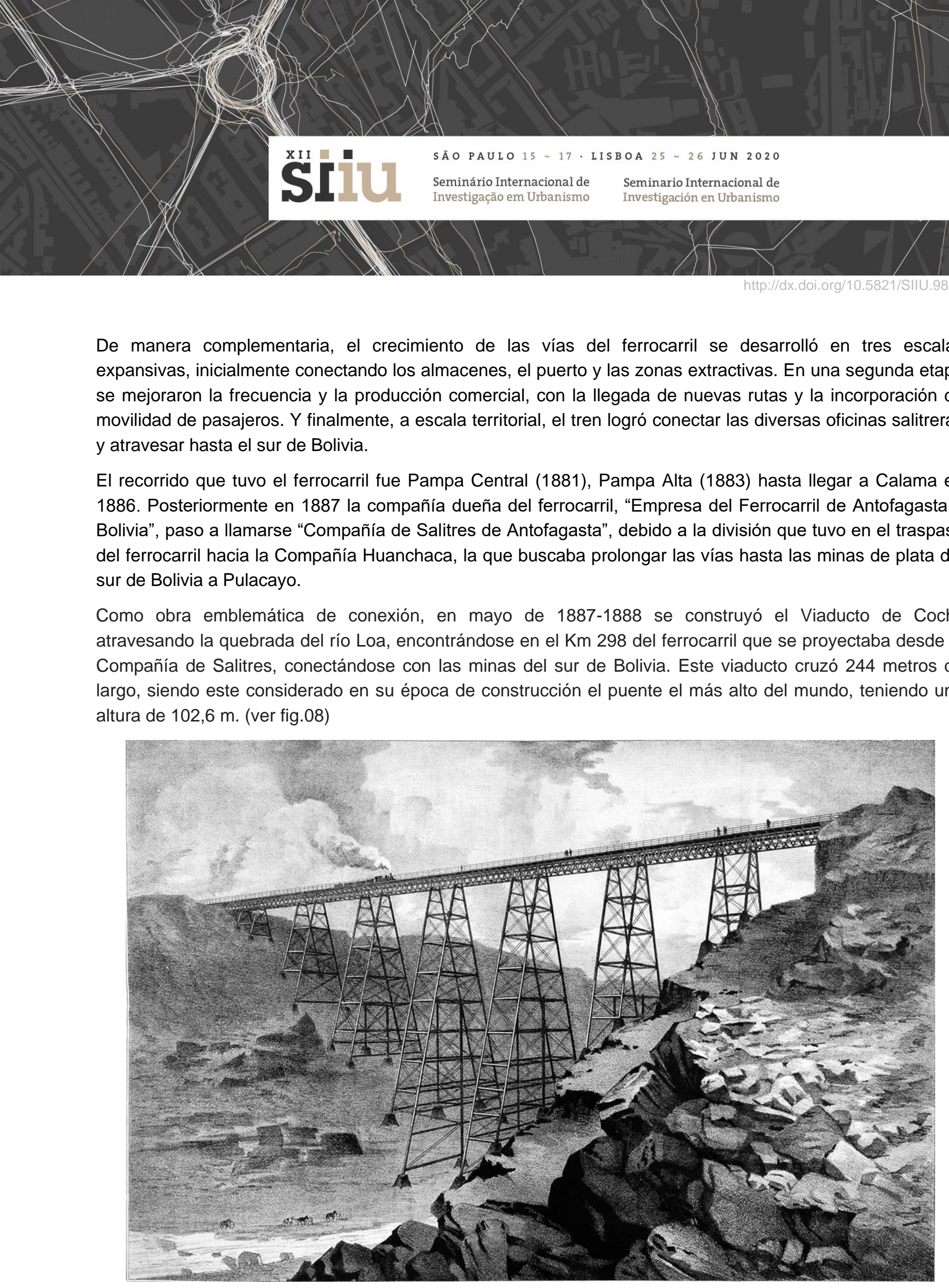

De manera complementaria, el crecimiento de las vías del ferrocarril se desarrolló en tres escalas expansivas, inicialmente conectando los almacenes, el puerto y las zonas extractivas. En una segunda etapa se mejoraron la frecuencia y la producción comercial, con la llegada de nuevas rutas y la incorporación de movilidad de pasajeros. Y finalmente, a escala territorial, el tren logró conectar las diversas oficinas salitreras

El recorrido que tuvo el ferrocarril fue Pampa Central (1881), Pampa Alta (1883) hasta llegar a Calama en 1886. Posteriormente en 1887 la compañía dueña del ferrocarril, "Empresa del Ferrocarril de Antofagasta a Bolivia", paso a llamarse "Compañía de Salitres de Antofagasta", debido a la división que tuvo en el traspaso del ferrocarril hacia la Compañía Huanchaca, la que buscaba prolongar las vías hasta las minas de plata del sur de Bolivia a Pulacayo.

Como obra emblemática de conexión, en mayo de 1887-1888 se construyó el Viaducto de Cochi, atravesando la quebrada del río Loa, encontrándose en el Km 298 del ferrocarril que se proyectaba desde la Compañía de Salitres, conectándose con las minas del sur de Bolivia. Este viaducto cruzó 244 metros de largo, siendo este considerado en su época de construcción el puente el más alto del mundo, teniendo una altura de 102,6 m. (ver fig.08)

Fig.8. Josiah Harding y Edward Woods, perspectiva del Viaducto de Conchi elaborada por Dudley Heath y publicada en The Engineer, 6 de abril de 1889. Fuente: Archivo Grace's Guide. 


\section{SÃOPAULO15 17 LISBOA $25 \sim 26$ JUN 2020}

Ese mismo mes la compañía Minería de Huanchaca, traspasó a una Compañía inglesa sus derechos, con la condición de una vez terminada la línea hasta el mineral, debía arrendarle a la empresa Huanchaca el ferrocarril por un plazo de diez años. The Antofagasta and Bolivia Railway Company Limited, fue el nombre con la que los accionistas ingleses denominaron esta nueva compañía. Harding, con experiencia en el diseño de las vías del tren y en el Viaducto de Conchi siguió a cargo de los estudios y construcción del ferrocarril de la línea hasta Pulacayo.

La llegada del ferrocarril hasta Uyuni el 25 en noviembre de 1889, y pronto hasta Pulacayo estuvo alineada con la idea de potenciar económicamente las zonas mineras del suroeste de Bolivia. El cruce de las fronteras del ferrocarril fue gestionado por parte de Aniceto Arce, quien fue presidente de Bolivia entre 1888-1892. Aniceto Arce supo sobreponerse a la presión política sobre los intereses económicos y prosperidad que esto traería al sector minero.

La conmoción y revuelo que produjo la llegada del tren a Oruro fue relevante en muchos aspectos, la primera que fue el anhelo que habían estado esperando los empresarios, por otro lado, el rechazo y miedo que esto generaba estaba involucrado directamente, a la Guerra del Pacífico por la nueva definición de límites entre ambos países.

Dicho esto, un diario de La Paz por la inauguración del ferrocarril en Oruro menciona:

"Las máquinas que penetraron a la Plaza de Oruro, tienen la siguiente inscripción: "Ferrocarril de Antofagasta a Bolivia". Esta inscripción equivale a decir que Chile está en posesión de Bolivia y que el potentado de Huanchaca es el protagonista de este drama. Ya no hay Bolivia, todo está consumado..." (Arce, 1930, p.161)

Dicho esto, las repercusiones sociales del "Fantasma del Ferrocarril", originaron retiradas de rieles, causando descarrilamientos, lo que sería un elemento de progreso económico, era un símbolo latente de conflicto y tensión en la primera etapa clave para la llegada del tren a suelo boliviano. Pero que lograrían borrar el éxito alcanzado por la maquina a vapor que recorrió y acortó el extenso desierto de Atacama. 


\section{SÃOPAULO15 17 LISBOA $25 \sim 26$ JUN 2020}

\section{Conclusiones}

El desierto de Atacama, ante de los avances de la Revolución industrial durante el siglo XIX, era visto como un lugar indómito, desafiante, con condiciones extremas, inalcanzable para cobijar la posibilidad de vida. $Y$ es en este contexto de adversidad, que los avances tecnológicos otorgaron nuevos saberes y desarrollos técnicos, que facilitaron al hombre el poder explorar y desarrollar nuevos asentamientos, en medio de un desierto dominado por la bastedad y la intensa radiación.

Y es a partir de este modelo económico, desierto como zona extractiva y puerto como lugar de distribución, que Antofagasta, en este rol como nodo central, adoptó cambios acelerados en el desarrollo industrial y los medios de transportes masivo. Que inició con la fundación y construcción de muelles hasta principio del siglo $\mathrm{XX}$, y que integró desde sus primeros años en 1873 al ferrocarril, como si se tratase de una verdadera extensión de los flujos navieros sobre el desierto, siendo años vitales para la construcción de equipamientos y progreso de la ciudad.

Sin embargo, estos medios de transportes no solo lograron ser el motor activo de la industria salitrera, sino que también fueron ordenadores de crecimientos urbano, en el que dividieron la retícula urbana, creando límites y transformaciones a diversas escalas, condicionando muchos cambios futuros que tuvo y tendría la ciudad a lo largo de su historia.

De acuerdo con lo planteado por Bird (1971) y reinterpretado por Rodríguez et al. (2006) en su modelo Anyport, Antofagasta vivió intensamente sus primeros años, dos grandes etapas de desarrollo, la primera iniciada en plena segunda ola de la Revolución industrial, más tardía que la descrita, pero que mantiene mayor parte de sus características, donde la ciudad y el puerto se desarrollan en torno a un núcleo central cívico, en el que se encontraban las casas comerciales y en el área norte la industria de Melbourne Clark, así como los equipamientos básicos. La segunda etapa de cambio se distingue con el crecimiento portuario y la llegada del ferrocarril, elemento propio de la Revolución industrial, que masificó el acelerado proceso de la industria portuaria y dividió y conectó al desierto con la costa, a través de las vías férreas llegando hasta la zona de Bolivia. Etapa de gran bonanza económica y crecimiento urbano que se extendió hasta inicio del siglo XX, donde habría un cambio radical en la industria de transporte en Antofagasta.

\section{BIBLIOGRAFÍA}

ACEVEDO, A. (1931). Antofagasta, la capital del desierto. En: Acevedo, Antonio. Croquis chileno (crónicas y relatos). (pp.65-77) .Santiago de Chile: Zig-Zag.

ALLIENDE, M. P., \& Piedad, M. (1993) Historia del ferrocarril en Chile. Santiago de Chile: Goethe InstitutPehuén.

ARANGO, S. (2013) Ciudad y Arquitectura. Seis generaciones que construyeron América Latina moderna, México: Fondo de Cultura Económica.

ARCE, I. (1997). Narraciones históricas de Antofagasta. Primera ed.: 1930. Antofagasta: s/e. 


\section{XII $\square$ SÃO PAULO $15 \sim 17 \cdot$ LISBOA $25 \sim 26$ JUN 2020

BERMÚDEZ, O. (1963). Historia del salitre, desde sus orígenes hasta la Guerra del Pacífico. Santiago: Ediciones de la Universidad de Chile.

ESCUDERO, E. y URZÚA, A. (1979). Inventario cartográfico de la mapoteca 'Luis Juan V. Abd-El-Kader' de la llustre Municipalidad de Antofagasta (carpetas no.4-7-11-12-13-14). Seminario superior para optar al título de profesor en Historia, Geografía y Educación Cívica, Universidad del Norte (Antofagasta).

ENRIQUE A.(ed.) (1979). Antofagasta la ciudad heroica. Aspectos históricos más sobresalientes de su vida y desarrollo, 1879-1979. Primer tomo. Antofagasta, Imprenta Agullo.

GALENO-Ibaceta, C. (2019) El Desierto de Atacama desde los artificios de la Revolución Industrial: reportes técnicos de ingeniería y arquitectura. Artículo no publicado.

GONZÁLEZ, J. A. (2008). La conquista de una frontera: Mentalidades y tecnologías en las vías de comunicación en el desierto de Atacama. En Revista de geografía Norte Grande (40), 23-46.

HARDOY, J. (1977). Las ciudades en América Latina. Seis ensayos sobre la urbanización contemporánea. (pp.134-148) Editorial: PAIDOS. Buenos Aires, Argentina.

MARIN, S. (1901) Estudios de los ferrocarriles chilenos. Publicado en los anales de la Universidad, editorial: imprenta Cervantes, Santiago, Chile.

OGNIRG (1924). Reminiscencias históricas de Antofagasta. El Mercurio de Antofagasta, 13 de febrero de 1924.8

ORTEGA, L. (1992) El proceso de industrialización en Chile 1850-1930. Historia. (26), pp. 213-246.

RODRIGUE, J. P; COMTOIS, C. y SLACK, B. (2006). Transport Terminals. En The geography of transport systems. (pp.126-133) London \& New York: Routledge.

The Engineer. (1889). The Antofagasta Railway, Bolivia. Viaduct over the river Loa. The highest viaduct in the world". En:The Engineer Magazine (abril 26): 346-48. 